\title{
Renoprotective effects of telmisartan after unilateral renal ablation in rats
}

This article was published in the following Dove Press journal: International Journal of Nephrology and Renovascular Disease 9 October 2013

Number of times this article has been viewed

Tomohiro Matsuo
Yasuyoshi Miyata
Yuji Sagara
Yoshikazu Higami
Shohei Tobu
Manabu Matsuo
Mitsuru Noguchi
Isao Shimokawa
Hiroshi Kanetake
Hideki Sakai
Department of Nephro-Urology,
Department of Investigative Pathology,
Nagasaki University Graduate School
of Biomedical Sciences, Nagasaki,
Japan

Correspondence: Yasuyoshi Miyata Department of Nephro-Urology, Nagasaki University School of Biomedical Sciences, I-7-I Sakamoto, Nagasaki 852-850I, Japan

$\mathrm{Tel}+8 \mid 958197340$

Fax +81958197343

Email int.doc.miya@m3.dion.ne.jp
Purpose: The renoprotective function of the angiotensin II type 1 receptor blocker (ARB) is well-known in various studies, including the animal model of renal failure. However, detailed temporal changes of pathological and molecular findings after unilateral nephrectomy are not fully understood. The main purpose of this study was to clarify the renoprotective effects and pathological changes induced by the ARB in rat-remnant kidney (RK) tissues after unilateral nephrectomy, but not after a 5/6 nephrectomy.

Methods: Telmisartan, which is structurally and functionally unique among ARB, was used in this study. Three rat groups were examined: A) no ARB administrated (RK, $n=21$ ); B) continuous subcutaneous infusion of an ARB administrated (RK-ARB, n=21); and C) a shamoperated group (Sham). Renal function was evaluated by blood urea nitrogen (BUN) levels and creatinine clearance (Ccr). Fibrosis was evaluated by hydroxyproline levels and Masson's trichrome staining. Expressions of angiotensin II type 1 receptor (AT1R) and transforming growth factor beta (TGF- $\beta$ ) were investigated by real-time polymerase chain reaction and Western blotting.

Results: There was no significant difference regarding body and kidney weight or pathological features evaluated by hematoxylin and eosin staining between the RK and RK-ARB groups. The Ccr in the RK group was significantly lower than that in the Sham group $(P<0.01)$, but no significant difference was found between the RK-ARB and Sham groups. The fibrotic area increased significantly with time after nephrectomy in the RK group. Although a similar trend was found in the RK-ARB group, the percentage of fibrous area in the RK-ARB group was significantly lower than that in the RK group at each time point $(P<0.01)$. AT1R mRNA levels in the RK group were regulated immediately compared with those in the RK-ARB group. Although expressions of the AT1R and TGF- $\beta$ were significantly higher in the RK-ARB group than in the Sham group, no significant differences were found between the RK-ARB and Sham group.

Conclusion: The ARB had renoprotective effects after unilateral nephrectomy. The ARB effectively maintained Ccr. Our results also showed the possibility that fibrotic changes mediated by AT1R and TGF- $\beta$ play an important role in renal protection. Moreover, this is the first report on changes of AT1R expression after using the ARB telmisartan in kidney tissues after unilateral nephrectomy. Finally, our results suggest that ARB may be useful to prevent renal failure in patients treated with nephrectomy.

Keywords: unilateral nephrectomy, telmisartan, angiotensin II type 1 receptor, renoprotection, fibrosis

\section{Introduction}

Radical nephrectomy is the gold-standard treatment for renal cell carcinoma (RCC). In addition, nephroureterectomy is the standard surgery of urothelial cancer of the 
upper urinary tract (UC-UUT). These urological cancers appear commonly in elderly individuals who often have renal dysfunction. Although partial nephrectomy is increasing to avoid renal dysfunction in patients with RCC, it is limited to small RCC. Furthermore, partial resection is not common for patients with UC-UUT. Thus, it is particularly important to preserve remnant kidney function after such unilateral nephrectomy. In addition to patients with RCC and UC-UUT, the preservation of unilateral kidney function is important in the living kidney donor population. ${ }^{1}$

There is general agreement that the renin-angiotensin system (RAS) helps to control blood pressure. In addition, the RAS plays important roles during the initiation and progression of various organ dysfunctions. ${ }^{2,3}$ In fact, the RAS inhibition has been shown to have a renoprotective effect, independent of blood pressure. ${ }^{4,5}$ Among the components of RAS, angiotensin II has been reported to be associated with renal function through its regulation of several physiological activities, such as the vasomotor tone, glomerular filtration, and accumulation of the extracellular matrix proteins; it also contributes to the progression of tissue inflammation. ${ }^{6,7,8}$ In addition, RAS also plays an important role in irreversible organ failure, including that of kidney, due to fibrosis. ${ }^{8}$ Thus, understanding the relationship between RAS and the molecular mechanism of fibrosis in kidney tissues is essential to determine the potential preventive strategies for renal function under pathological conditions.

Angiotensin II can reach all accessible target organs to exert its biological and pathological effects by activating its receptors, ie, the angiotensin II type 1 receptors (AT1R) and the angiotensin II type 2 receptors (AT2R). Although the function of AT2R is not fully understood, many studies have shown that the pathophysiological effects of tissues are mainly mediated via binding to AT1R. ${ }^{9}$ In fact, AT1R blockade ARB was found to be effective not only for lowering blood pressure, but it was also found to be effective in protecting against remodeling and functionally preserving the kidney. ${ }^{10}$ In addition, clinical evidence has demonstrated that ARB could improve renal function or slow disease progression in nephropathy. ${ }^{11}$ Thus, information regarding the relationship between ARB and AT1R is important to understand the detailed mechanisms involved in this renoprotective effect.

Many reports have been published regarding the relationship between ARBs and their renoprotective function in vivo. However, most reports have focused on the chronic kidney disease that occurs with hypertension and diabetes mellitus. On the other hand, several reports have shown the renoprotective effects of ARBs and/or AT1Rs in acute renal failure by using a subtotal of nephrectomized animal models. ${ }^{12,13}$ However, this situation is not common under clinical conditions. Conversely, despite the fact that unilateral nephrectomy is the standard surgery for a variety of urological cancers and transplantation, there is little detailed information on the renoprotective mechanisms of ARB in such pathological conditions in vivo.

The purpose of this study was to clarify the renoprotective effects of ARB after the unilateral nephrectomy in animal models that fit into the reality of clinical situations. In addition, changes of the AT1R mRNA and protein expression were investigated in a similar model. We also examined the relationship between the AT1 expression and hydroxyproline (Hyp), a fibrotic marker, and transforming growth factor beta (TGF- $\beta$ ), a representative renal fibrosis-related molecule, in unilateral nephrectomized rat kidney tissues treated with ARB.

\section{Methods}

\section{Animal models}

Twelve-week-old male F344 rats ( $n=70$; Charles River Laboratories, Inc, Yokohama, Japan) were used. Under pentobarbital sodium anesthesia ( $50 \mathrm{mg} / \mathrm{kg}$ intraperitoneally), the left kidney was removed. The removed kidney was dissected and prepared for histology, immunohistochemical study, Western blotting, and a real-time reverse transcriptionpolymerase chain reaction (RT-PCR). Samples for Western blotting and RT-PCR were snap-frozen in liquid nitrogen and stored at $-80^{\circ} \mathrm{C}$. The muscle and skin layers were closed after subcutaneous implantation of osmotic minipumps (Alzet model 2004, DURECT Corporation, Cupertino, CA, USA) filled with dimethyl sulfoxide or telmisartan (Nippon Boehringer Ingelheim Co, Ltd, Tokyo, Japan).

Twenty-one rats underwent anesthesia and manipulation of the renal pedicles without removal of any renal mass (Sham group). These rats were housed in individual cages in a temperature-controlled room at $22^{\circ} \mathrm{C}$ and a 12 -hour light/dark cycle (Laboratory Animal Center for Biomedical Research, Nagasaki University School of Medicine, Nagasaki, Japan), fed a standard diet, and allowed to drink tap water ad libitum. All procedures were performed according to the protocols approved by the Animal Care and Use Committee of Nagasaki University.

\section{Experimental rat groups}

A total of 42 nephrectomized rats were divided into two groups: the A) renal ablation group treated with physiological 
saline starting on the day of surgery $(\mathrm{RK} ; \mathrm{n}=21)$ and the B) renal ablation group treated with $1 \mathrm{mg} / \mathrm{kg} /$ day telmisartan, starting on the day of surgery $(R K-A R B ; n=21)$. Seven rats from each of the two groups were examined 2, 4, and 8 weeks after the operation. The minipumps were exchanged every 4 weeks. After observation, the right kidney was removed and processed, as described in the Animal models. This was considered the control kidney. All the rats were maintained under similar housing/feeding conditions as the sham rats. The sham rats were not treated with telmisartan during the research period.

\section{Blood urea nitrogen levels and creatinine clearance in blood and urine}

Renal function was evaluated by blood urea nitrogen (BUN) levels and creatinine clearance (Ccr). Blood samples were obtained from the tail vein before the operation and at the end of treatment. Serum was obtained by centrifuging blood samples at 3,000 rpm for 20 minutes at $4^{\circ} \mathrm{C}$. For urine samples, the rats were placed in individual cages for 24 hours. After the urine volume was measured, samples were collected and centrifuged at 3,000 rpm for 10 minutes at $4{ }^{\circ} \mathrm{C}$. The supernatants were used for the creatinine concentration assay. Blood and urine samples were stored at $-20^{\circ} \mathrm{C}$ until the assay. Each $\mathrm{Ccr}$ value was measured using enzyme-linked immunoassay kits (Cayman Chemical Company, Ann Arbor, MI, USA). The BUN was measured using the Wako BUN-test (Wako Pure Chemical Industries, Ltd, Osaka, Japan); the absorbance was detected at $570 \mathrm{~nm}$ for BUN and at $492 \mathrm{~nm}$ for creatinine, using a microplate reader (Thermo/LabSystems Multiskan RC, Artisan Technology Group ${ }^{\circledR}$, Champaign, IL, USA).

\section{Measurement of Hyp}

Hyp was evaluated according to previous reports. ${ }^{14,15}$ The tissue homogenate was hydrolyzed in alkali. The hydrolyzed sample was mixed with a buffered chloramine-T reagent, and oxidation was allowed to proceed for 25 minutes at room temperature. The chromophore was developed with the addition of an Ehrlich's reagent. The absorbance was read at $550 \mathrm{~nm}$ using an absorption spectrophotometer (Thermo/ LabSystems Multiskan RC).

\section{Histological assessment}

At 2, 4, and 8 weeks after the left nephrectomy or sham operation, the rats were sacrificed. The remnant kidneys were removed, fixed in $10 \%$ buffered formalin, and embedded in paraffin. Subsequently, $4 \mu \mathrm{m}$-thick sections were stained with hematoxylin and eosin (H\&E) stain and Masson's trichrome (MT) stain for light microscopy. Quantitative data for percentage of fibrotic area were measured in three to six different fields per MT-stained specimen. Slides were evaluated twice at different times by two investigators (TM and YS).

\section{RNA extraction and quantitative analysis of AT I R using RT-PCR}

The expression of AT1R mRNA in the renal cortex was assessed by RT-PCR, as described previously. ${ }^{16}$ The kidney was preserved with RNAlater ${ }^{\mathrm{TM}}$ (Ambion, Inc, Austin, TX, USA) for RT-PCR and frozen at $-80^{\circ} \mathrm{C}$. Frozen renal cortex (20-30 mg) was homogenized in $1 \mathrm{~mL}$ of QIAzol reagent (QIAGEN Inc, Valencia, CA, USA) using a polytron homogenizer for 30 seconds. For RT-PCR, RNA was extracted from the kidney cortex using an RNeasy Mini Kit (QIAGEN), and it was stored at $-80^{\circ} \mathrm{C}$. The AT1R gene expression was analyzed by real-time quantitative RT-PCR performed using the TaqMan system on the basis of real-time detection of accumulated fluorescence (ABI Prism 7700; PerkinElmer, Waltham, MA, USA). ${ }^{17}$ Prevalidated primers and probes for the AT1a receptor (Rn00578456_m1) were obtained via Assays-on-Demand (Applied Biosystems, product line of Life Technologies, Carlsbad, CA, USA). Total RNA yields were determined by spectrophotometry. The amplification was performed using the method of Cao et al. ${ }^{18}$

\section{Protein extraction and Western blotting for AT IR and TGF- $\beta$}

Protein levels of the AT1R in the renal tissues were analyzed by Western blotting, as previously described. ${ }^{15,19,20}$ The membrane was reacted with a polyclonal anti-AT1 receptor antibody $(1: 1,000$; Santa Cruz Biotechnology Inc, Santa Cruz, CA, USA), followed by incubation with a horseradish peroxidase-conjugated secondary antibody (for the AT1 receptor: goat antirabbit IgG, 1:1,000; Santa Cruz Biotechnology Inc, Santa Cruz, CA, USA). To check for equal loading, membranes were reprobed with an antibody against $\beta$-actin (Sigma Chemicals, Perth, WA, Australia).

\section{Statistical analysis}

Results were expressed as mean \pm standard error of mean. Statistical comparisons were made by analysis of variance, followed by the Kruskal-Wallis test. All statistical analyses were two-sided, and significance was defined as a $P$ value of $<0.05$. All statistical analyses were performed on a personal computer with the statistical package StatView 
for Windows (version 5.0, Abacus Concept, Inc, Berkeley, CA, USA).

\section{Results}

\section{Changes of renal function and fibrosis}

We compared kidney weight (KW), body weight (BW), and its ratio $(\mathrm{KW} / \mathrm{BW})$ in all groups. At baseline, there was no significant difference in any parameters among the groups. At 2 weeks, the KW/BW of the RK $(3.89 \pm 0.40)$ and the RK-ARB (3.76 \pm 0.23$)$ groups was significantly higher than that of the Sham group $(3.37 \pm 0.18, P<0.01)$. However, there was no significant difference between the two nephrectomized groups $(P=0.07)$. At 8 weeks, although $\mathrm{KW} / \mathrm{BW}$ in the RK-ARB group $(3.99 \pm 0.27)$ tended to be lower than that in the RK group $(4.16 \pm 0.26)$, this difference was not significant $(P=0.10)$. Finally, there was no significant difference in $\mathrm{BW}$ and $\mathrm{KW}$ between these two groups at any time point.

Changes in the BUN levels are shown in Figure 1A. At 2 weeks, significant increases of the BUN levels compared with the baseline were observed in both the RK and the RKARB groups. On the other hand, no significant differences were noted at the other time points in either the RK or the RK-ARB groups.

Next, we examined changes in Ccr (Figure 1B). Ccr in the RK group decreased significantly after 2 weeks when compared with that in the Sham group $(P<0.01)$ and, after 4 weeks, compared with that in the RK-ARB group $(P<0.01)$. However, although $\mathrm{Ccr}$ in the RK-ARB group had a trend to be lower than in the Sham group at 2 and 4 weeks, the differences were not significant $(P=0.73$ and $P=0.23$, respectively).

Changes in the Hyp levels are shown in Figure 1C. Progressive increases in the Hyp levels were detected in the RK group. Such increases were significant when compared with the baseline after 2 weeks. In addition, the Hyp levels in the RK group were significantly higher $(P<0.01)$ than those at the corresponding time point in the RK-ARB group from 4 weeks after nephrectomy (Figure 1C).

\section{Histopathological changes}

Figure 2A-C show H\&E-stained samples. However, there was no significant change in the renal tissue in any of the three rat models. The representative examples of MT staining and the percentage of fibrotic area in rat tissues are shown in Figure 2D-J, respectively. At first, we examined control kidney tissues at the baseline (Figure 2D) and at 8 weeks (Figure 2E). We noticed no differences between
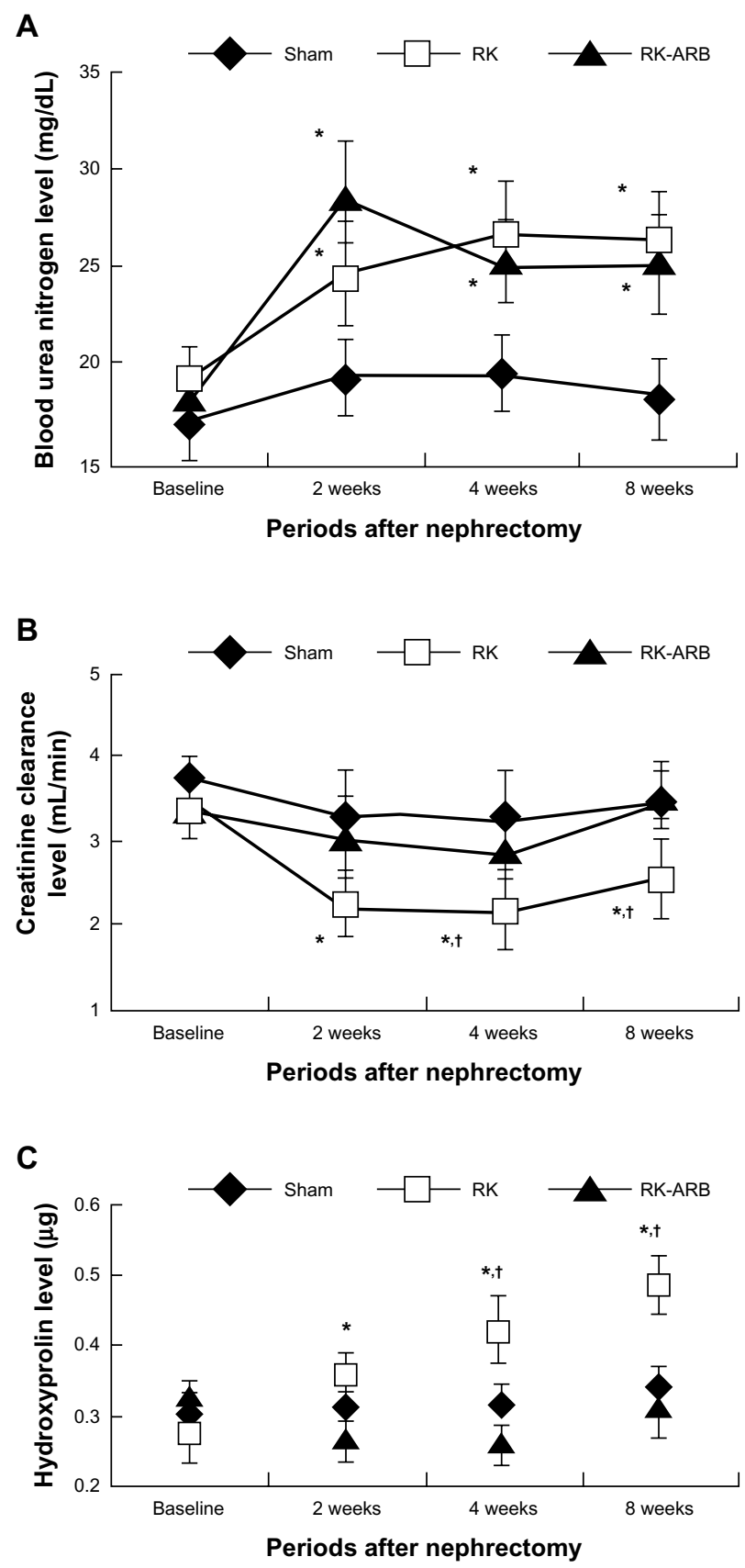

Figure I Course of changes in BUN, Ccr, and Hyp levels in the three rat groups. Notes: Figure I (A-C) showed the time course of changes in BUN, Ccr, and Hyp levels in the three rat groups. ${ }^{*} P<0.05$ versus Sham group rats at the same time. $+P<0.05$ versus $R K-A R B$ at the same time.

Abbreviations: RK, rat-remnant kidney; ARB, angiotensin II type I receptor blocker; BUN, blood urea nitrogen; Ccr, creatinine clearance; Hyp, hydroxyproline; Sham, sham-operated group.

the control tissues in any specimens. Figure $2 \mathrm{~F}$ and $\mathrm{G}$ are representative examples of MT-stained sections in the RK group at 2 and 8 weeks, respectively. The fibrous area in the RK group at 2 weeks $(20.8 \% \pm 4.9 \%$, Figure $2 \mathrm{~F})$ was larger $(P<0.01)$ than that in the Sham group, including those at 8 weeks $(10.8 \% \pm 2.0 \%$, Figure $2 \mathrm{D})$. In addition, fibrosis in the RK group at 8 weeks $(53.3 \% \pm 5.2 \%$, Figure $2 \mathrm{G})$ 

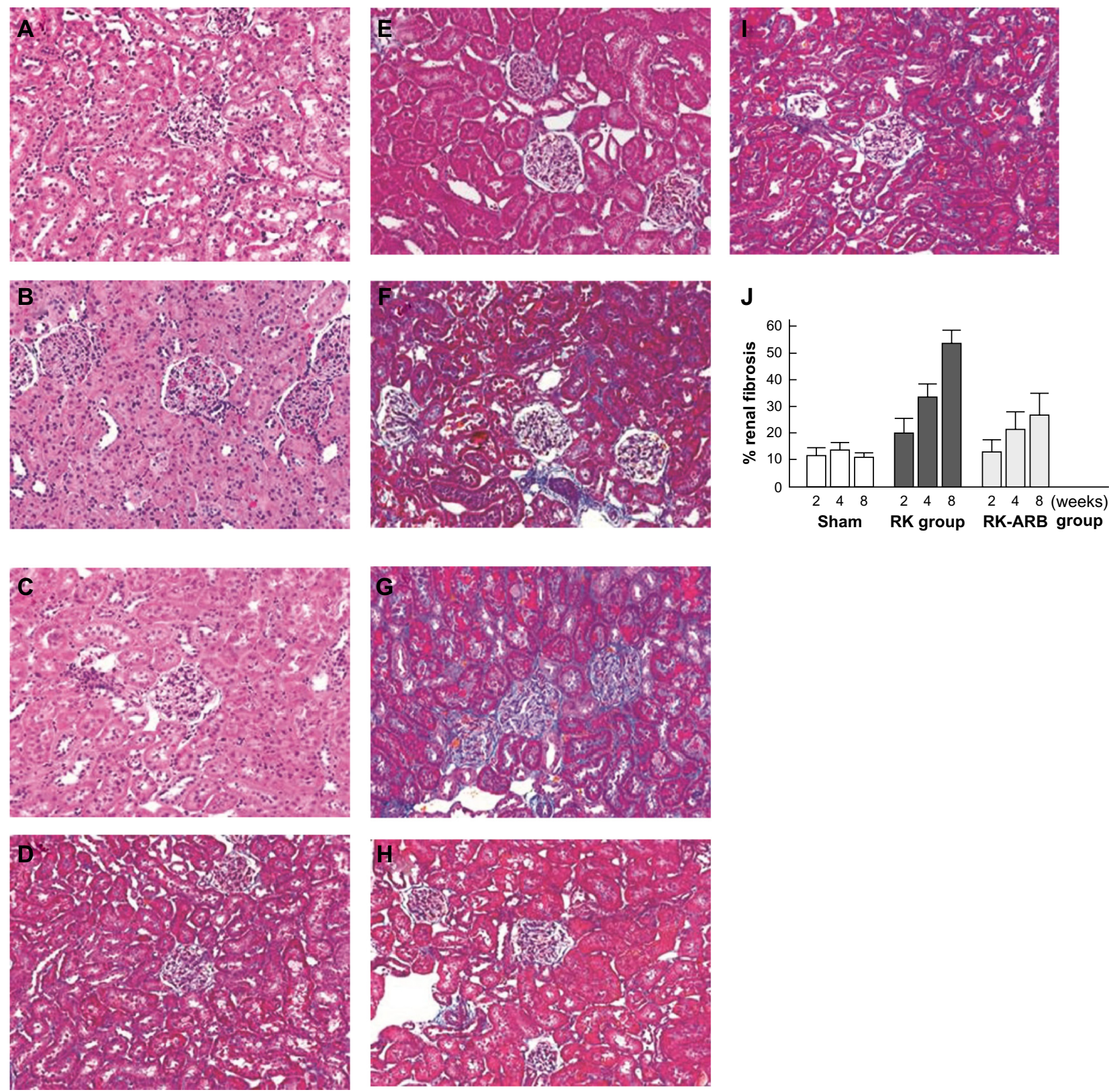

Figure 2 (A-C) show a section stained with H\&E staining.

Notes: There were no changes in the glomeruli or renal tubules in any of the three rat groups after 8 weeks - (A) Sham group; (B) RK group; and (C) RK-ARB group. Figure 2 (D-I) show representative examples of MT staining. Control kidney tissues at baseline and at 8 weeks are shown in Figure $2 \mathbf{D}$ and $\mathbf{E}$, respectively. Fibrosis in the RK group at 2 weeks (Figure $2 \mathbf{F}$ ) was significantly different $(P<0.0 \mathrm{I})$, compared with that in the RK groups at 8 weeks (Figure $2 \mathbf{G}$ ). Figure $2 \mathbf{H}$ and $\mathbf{I}$ show representative specimens in the RK-ARB at 2 weeks and at 8 weeks, respectively. (Original magnification, $\times 200$ ). The percentage of fibrous area in the RK-ARB group was significantly lower $(P<0.0 \mathrm{I})$ than that in the RK group at each week (Figure 2J).

Abbreviations: RK, rat-remnant kidney; ARB, angiotensin II type I receptor blocker; MT, Masson's trichrome; H\&E, hematoxylin and eosin; sham, sham-operated group.

was significantly different $(P<0.01)$ compared with that in the Sham and the RK groups at 2 weeks (Figure 2F). In particular, marked sclerosis of the glomeruli was observed in the RK group at 8 weeks (Figure $2 \mathrm{G}$ ). Thus, the fibrotic area increased considerably with time after nephrectomy in the RK group. Although a similar trend was also seen in the RK-ARB group at 2 weeks (Figure 2H) and at 8 weeks (Figure 2I), the percentage of fibrous area in the RK-ARB group was significantly lower than that in the RK group at each week (Figure 2J).

\section{Altered $\mathrm{mRNA}$ and protein levels of AT IR}

Changes in the mRNA expression of AT1R are shown in Figure 3. In the RK group, AT1R was upregulated immediately compared with the RK-ARB group. At 2 weeks, the 


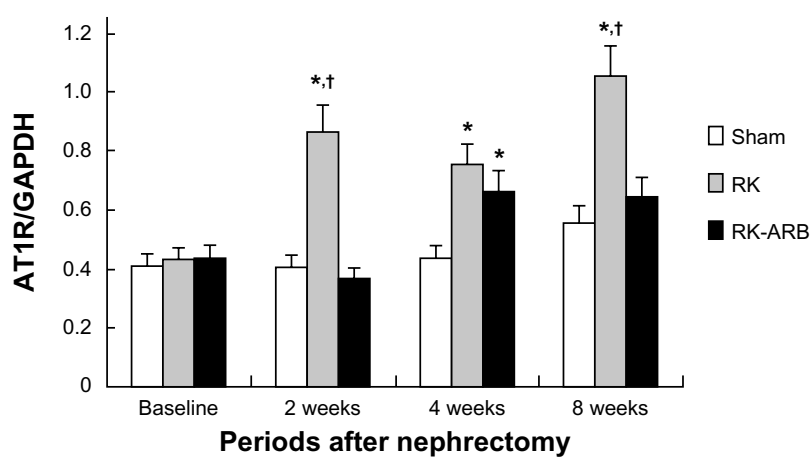

Figure 3 Altered ATIR mRNA expression levels.

Notes: Significant increases in the ATIR mRNA expression were observed 2 weeks after the first operation in the RK group. Although ATIR mRNA expression level in the RK-ARB group was higher than that in the Sham group at 4 weeks, the expression was suppressed in the RK-ARB group at 8 weeks, and no significant difference was found between the Sham and RK-ARB groups at 8 weeks. ${ }^{*} P<0.05$ versus the Sham group. ${ }^{\dagger}<0.05$ versus the RK-ARB group.

Abbreviations: ATIR, angiotensin II type I receptor; GAPDH, glyceraldehyde 3-phosphate dehydrogenase; RK, rat-remnant kidney; ARB, angiotensin II type I receptor blocker; sham, sham-operated group.

AT1R mRNA expression in the RK group was significantly higher $(P<0.01)$ than that in the Sham and the RK-ARB groups (Figure 3 ). In addition, such increases compared with the Sham group were noted for all time points. On the other hand, in the RK-ARB group, mRNA expression showed significant differences compared with the Sham group at 4 weeks $(P<0.01)$. However, unlike the RK group, significant differences between the RK-ARB and Sham groups were not detected at 8 weeks.

Representative examples of the Western blotting regarding AT1R expression are shown in Figure 4A. Increased AT1R expression compared with the Sham group was found at all time points for the RK group, and the AT1R expression levels increased with time. On the other hand, such expression was not found in the RK-ARB group at any time point.

\section{Altered protein levels of TGF- $\beta$}

The TGF- $\beta$ expression is shown in Figure 4B. In the RK group, increases in the TGF- $\beta$ expression were noted from 2 weeks after surgery. However, increasing TGF- $\beta$ expression with time was not found in the RK group. On the other hand, in the RK-ARB group, low TGF- $\beta$ expression was found at all time points, and all of the TGF- $\beta$ expression levels were similar. When the correlation between the AT1R expression and the TGF- $\beta$ expression was analyzed using relative densitometric levels of each protein per unit of $\beta$-actin, no significant correlation was detected. Similarly, we found no significant relationship between the AT1R gene expression and the TGF- $\beta$ expression (Figures 3 and 4B).
A

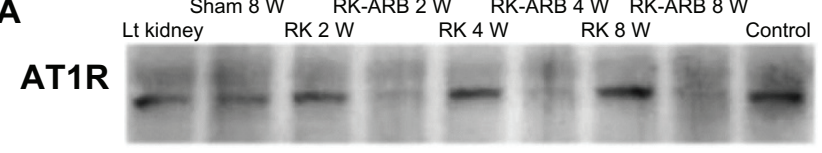

$\beta$-actin

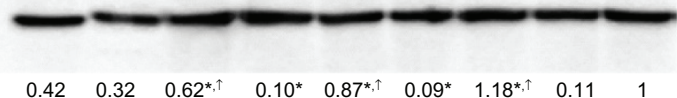

B \begin{tabular}{lllll}
\multicolumn{1}{c}{ Sham $8 \mathrm{~W}$} & RK-ARB $2 \mathrm{~W}$ & RK-ARB $4 \mathrm{~W}$ & RK-ARB $8 \mathrm{~W}$ \\
Lt kidney & RK $2 \mathrm{~W}$ & RK $4 \mathrm{~W}$ & RK 8 W &
\end{tabular}

TGF- $\beta$

$\beta$-actin

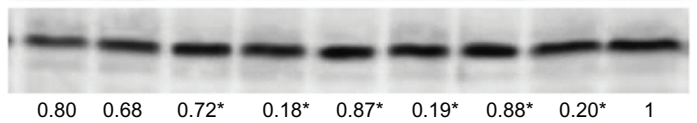

Figure 4 Altered protein levels of ATIR.

Notes: Western blotting revealed ATIR expression in the RK group was higher than in the Sham and the RK-ARB groups. Numbers represent relative densitometric levels of ATIR per unit $\beta$-actin in the pellets. ${ }^{*} P<0.0$ I RK versus RK-ARB at 2 weeks, 4 weeks, and 8 weeks; ${ }^{\dagger} P<0.05$ RK at 2 weeks versus RK at 4 weeks; RK at 2 weeks versus RK at 8 weeks; and RK at 4 weeks versus RK at 8 weeks. Figure 4B shows the altered TGF- $\beta$ protein levels. In the RK group, significant increases in the TGF- $\beta$ expression were observed at 2 weeks after the first operation. Numbers represent relative densitometric levels of TGF- $\beta$ per unit $\beta$-actin in the pellets. $* P<0.0$ I RK at 2 weeks versus RK-ARB at 2 weeks; RK at 4 weeks versus RK-ARB at 4 weeks; and RK at 8 weeks versus RK-ARB at 8 weeks.

Abbreviations: ATIR, angiotensin II type I receptor; Lt, left; RK, rat-remnant kidney; ARB, angiotensin II type I receptor blocker; TGF- $\beta$, transforming growth factor beta; w, weeks; sham, sham-operated group.

\section{Discussion}

Many investigators have reported that blockage of the RAS by ARBs could maintain renal function in various types of nephropathy. ${ }^{12,13,21}$ In addition to examining chronic renal failure progression, a study used 5/6 nephrectomized rat models to clarify the renoprotective effects of ARBs in acute renal failure. ${ }^{22}$ In contrast, this is the first report regarding changes of renal function and pathological features induced by ARBs under unilateral nephrectomized conditions that do not represent renal failure.

The present study detected that the use of telmisartan resulted in a decrease in AT1R mRNA and protein expression. AT1R is known to mediate most of the physiological and pathological effects of angiotensin II, such as hyperdynamic actions, endocrine functions, and mitogenic effects in the kidney. ${ }^{23-26}$ Thus, understanding the mechanism of the AT1R expression in kidney tissues under pathological conditions is important to discuss potential strategies to maintain renal function. With regard to the relationship between the AT1R and ARBs, there is a report that the AT1R expression was suppressed by telmisartan at both the mRNA and the protein levels in vascular smooth muscle cells. ${ }^{27}$ However, to our knowledge, 
this is the first report on changes of the AT1R expression after using telmisartan in kidney tissues under pathological conditions. Our results showed that both the expression of AT1R mRNA and protein in the RK-ARB group were significantly lower than those in the RK group. On the basis of these facts, we speculate that the suppression of AT1R expression is an important mechanism underlying the renoprotective effect of telmisartan. On the other hand, telmisartan also induces peroxisome proliferator-activated receptor gamma (PPAR $\gamma$ ). Furthermore, PPAR $\gamma$ is associated with fibrosis under a variety of conditions. ${ }^{28}$ Therefore, in addition to AT1R-related activities, investigations regarding the change of PPAR $\gamma$-related activation, including the regulation of fibrosis under unilateral nephrectomized conditions, are necessary.

Another interesting finding of this study was that TGF- $\beta$ expression in the RK-ARB group was lower than that in the RK group. TGF- $\beta$ has been shown to mediate various key renal pathological events, including fibrosis, during loss of renal function. ${ }^{29}$ In addition, TGF- $\beta$ also induces autophagy and apoptosis, leading to fibrosis, in renal tissues. ${ }^{30}$ Thus suppression of the TGF- $\beta$ expression leads to protection of renal function. We speculate that telmisartan may protect renal function via the regulation of some of these mechanisms. Our results also showed that the Hyp expression, a marker of fibrosis, was suppressed by telmisartan. Interestingly, AT1R was reported to be a regulator of TGF- $\beta$ in nephropathy. ${ }^{31}$ From these facts, we hypothesized that suppressed AT1R expression by telmisartan would lead to the inhibition of TGF- $\beta$ expression in this renal dysfunction model. Unfortunately, our results did not show a significant correlation between the TGF- $\beta$ expression and the AT1R expression. Thus, it is likely that the TGF- $\beta$ expression in kidney tissues is regulated by other factors under pathological conditions.

Our study showed that telmisartan was effective in maintaining Ccr. On the other hand, pathological features evaluated by H\&E staining showed no differences between the RK and the RK-ARB groups. The unilateral nephrectomy model is thought to result in relatively mild damage to the remnant kidney compared with the $5 / 6$ nephrectomy model..$^{22}$ In recent years, partial nephrectomy has been recommended for patients with low-stage RCC to maintain renal function. Our results support this strategy from the renoprotective perspective.

In this study, we used telmisartan, which is structurally and functionally unique among ARBs. Although most ARBs are biphenyl-tetrazole derivatives, telmisartan is a biphenyl-nontetrazole derivative and has greater lipophilicity and a longer half-life. In addition, interestingly, the antiinflammatory and fibrous effects of telmisartan are the result of the PPAR $\gamma$ activation. These characteristics favor the renoprotective effect. Several reports have shown that telmisartan has better renal protective effects than other ARBs in nephropathy. ${ }^{21,32}$ Further studies are necessary to determine whether such findings are common with other ARBs. Detailed investigations and a good understanding of the renoprotective function of ARBs are essential for the treatment and observation of chronic kidney disease. ${ }^{33}$

One of the major limitations of this study is that we used only a single ARB, ie, telmisartan. Therefore, no direct comparison within this study regarding the general effects of ARBs could be made. Another limitation is the lack of data regarding blood pressure. Telmisartan suppressed blood pressure in various animal models with renal dysfunction. It is possible that this also occurred in our study. On the other hand, we have some evidence that telmisartan may cause symptomatic hypotension in patients with nephrectomy (data not shown). A further limitation is that our results showed no significant difference on renal function when evaluated by BUN levels and Ccr. In addition, we have no data regarding other functional parameters affected by fibrosis. Although we speculated that the renoprotective function of telmisartan might be undervalued in the relatively early phase after nephrectomy, this study has little evidence to confirm this. Thus, more detailed clinical studies and pathological examinations, including the renoprotective and hypotensive function of telmisartan after unilateral ablation, are necessary.

\section{Conclusion}

It can be concluded that the ARB telmisartan protected renal function after nephrectomy in a rat model. This conforms to the preexisting clinical evidence. In terms of the mechanism of the renoprotective effects, inhibition of fibrosis was important, and AT1R and TGF- $\beta$ are speculated to be important in the regulation of fibrotic changes.

\section{Acknowledgment}

We are grateful to Takumi Shimogama for his support.

\section{Disclosure}

The authors report no conflicts of interest in this work. This study was not supported financially by any funding agency or company.

\section{References}

1. Martin FL, McKie PM, Cataliotti A, et al. Experimental mild renal insufficiency mediates early cardiac apoptosis, fibrosis, and diastolic dysfunction: a kidney-heart connection. Am J Physiol Regul Integr Comp Physiol. 2012;302(2):R292-R299. 
2. Szymanski MK, Damman K, van Veldhuisen DJ, van Gilst WH, Hillege HL, de Boer RA. Prognostic value of renin and prorenin in heart failure patients with decreased kidney function. Am Heart J. 2011;162(3): 487-493.

3. Parvanova A, Chiurchiu C, Ruggenenti P, Remuzzi G. Inhibition of the renin-angiotensin system and cardio-renal protection: focus on losartan and angiotensin receptor blockade. Expert Opin Pharmacother. 2005;6(11):1931-1942.

4. Shiga Microalbuminuria Reduction Trial (SMART) Group, Uzu T, Sawaguchi M, Maegawa H, Kashiwagi A. Reduction of microalbuminuria in patients with type 2 diabetes: the Shiga Microalbuminuria Reduction Trial (SMART). Diabetes Care. 2007;30(6):1581-1583.

5. Koike H, Sada T, Mizuno M. In vitro and in vivo pharmacology of olmesartan medoxomil, an angiotensin II type AT1 receptor antagonist. J Hypertens Suppl. 2001;19(1):S3-S14.

6. Lavoz C, Rodrigues-Diez R, Benito-Martin A, et al. Angiotensin II contributes to renal fibrosis independently of Notch pathway activation. PLoS One. 2012;7(7):e40490.

7. Kagami S, Border WA, Miller DE, Noble NA. Angiotensin II stimulates extracellular matrix protein synthesis through induction of transforming growth factor-beta expression in rat glomerular mesangial cells. J Clin Invest. 1994;93(6):2431-2437.

8. Remuzzi G, Perico N, Macia M, Ruggenenti P. The role of reninangiotensin-aldosterone system in the progression of chronic kidney disease. Kidney Int Suppl. 2005;99:S57-S65.

9. Takeuchi Y, Yamauchi K, Nakamura J, Shigematsu S, Hashizume K. Angiotensin II regulates migration in mouse cultured mesangial cells: evidence for the presence of receptor subtype-specific regulation. J Endocrinol. 2006;191(2):361-367.

10. Mandarim-de-Lacerda CA, Pereira LM. Effect of telmisartan on preexistent cardiac and renal lesions in spontaneously hypertensive mature rats. Histrol Histpathol. 2004;19(3):727-733.

11. Banki NF, Ver A, Wagner LJ, et al. Aldosterone antagonists in monotherapy are protective against streptozotocin-induced diabetic nephropathy in rats. PLoS One. 2012;7(6):e39938.

12. Toba H, Tojo C, Wang J, Noda K, Kobara M, Nakata T. Telmisartan inhibits vascular dysfunction and inflammation via activation of peroxisome proliferator-activated receptor- $\gamma$ in subtotal nephrectomized rat. Eur J Pharmacol. 2012;685(1-3):91-98.

13. Fujimoto S, Satoh M, Horike H, et al. Olmesartan ameliorates progressive glomerular injury in subtotal nephrectomized rats through suppression of superoxide production. Hypertens Res. 2008;31(2):305-313.

14. Varghese Z, Moorhead JF, Wills MR. Plasma hydroxyproline fractions in patients with dialysis osteodystrophy. Clin Chim Acta. 1981;110(1): 105-111.

15. Reddy GK, Enwemeka CS. A simplified method for the analysis of hydroxyproline in biological tissues. Clin Biochem. 1996;29(3): 225-229.

16. Rodrigo MC, Martin DS, Redetzke RA, Eyster KM. A method for the extraction of high quality RNA and protein from single small samples of arteries and veins preserved in RNAlater. $J$ Pharmacol Toxicol Methods. 2002;47(2):87-92.

17. Bonnet F, Cooper ME, Carey RM, Casley D, Cao Z. Vascular expression of angiotensin type 2 receptor in the adult rat: Influence of angiotensin II infusion. J Hypertens. 2001;19(6):1075-1081.
18. Cao Z, Bonnet F, Candido R, et al. Angiotensin type 2 receptor antagonism confers renal protection in a rat model of progressive renal injury. J Am Soc Nephrol. 2002;13(7):1773-1787.

19. Yao L, Kobori H, Rahman M, et al. Olmesartan improves endothelininduced hypertension and oxidative stress in rats. Hypertens Res. 2004;27(7):493-500.

20. Nishiyama A, Yoshizumi M, Rahman M, et al. Effects of AT1 receptor blockade on renal injury and mitogen-activated protein activity in Dahl salt-sensitive rats. Kidney Int. 2004;65(3):972-981.

21. Bakris G, Burgess E, Weir M, Davidai G, Koval S; AMADEO Study Investigators. Telmisartan is more effective than losartan in reducing proteinuria in patients with diabetic nephropathy. Kidney Int. 2008;74(3):364-369.

22. Nagai Y, Yao, L, Kobori H, et al. Temporary angiotensin II blockade at the prediabetic stage attenuates the development of renal injury in type 2 diabetic rats. J Am Soc Nephrol. 2005;16(3):703-711.

23. Goodfriend TL, Elliott ME, Catt KJ. Angiotensin receptors and their antagonists. N Engl J Med. 1996;334(25):1649-1654.

24. Unger T, Culman J, Gohlke P. Angiotensin II receptor blockade and endorgan protection: pharmacological rationale and evidence. J Hypertens Suppl. 1998;16(7):S3-S9.

25. Duncan JA, Scholey JW, Miller JA. Angiotensin II type 1 receptor gene polymorphisms in humans: physiology and pathophysiology of the genotypes. Curr Opin Nephrol Hypertens. 2001;10(1): 111-116.

26. Siragy HM, Carey RM. Angiotensin type 2 receptors: potential importance in the regulation of blood pressure. Curr Opin Nephrol Hypertens. 2001;10(1):99-103.

27. Imayama I, Ichiki $\mathrm{T}$, Inanaga $\mathrm{K}$, et al. Telmisartan downregulates angiotensin II type 1 receptor through activation of peroxisome proliferator-activated receptor gamma. Cardiovasc Res. 2006;72(1): 184-190.

28. Attia YM, Elalkamy EF, Hammam OA, Mahmoud SS, El-Khatib AS. Telmisartan, an AT1 receptor blocker and a PPAR gamma activator, alleviates liver fibrosis induced experimentally by Schistosoma mansoni infection. Parasit Vectors. 2013;6:199.

29. López-Hernández FJ, López-Novoa JM. Role of TGF- $\beta$ in chronic kidney disease: an integration of tubular, glomerular and vascular effects. Cell Tissue Res. 2012;347(1):141-154.

30. Xu Y, Yang S, Huang J, Ruan S, Zheng Z, Lin J. Tgf- $\beta 1$ induces autophagy and promotes apoptosis in renal tubular epithelial cells. Int J Mol Med. 2012;29(5):781-790.

31. Koshizaka M, Takemoto M, Sato S, et al. An angiotensin II type 1 receptor blocker prevents renal injury via inhibition of the Notch pathway in Ins2 Akita diabetic mice. Exp Diabetes Res. 2012;2012:159874.

32. Khan AH, Imig JD. Telmisartan provides better renal protection than valsartan in a rat model of metabolic syndrome. Am J Hypertens. 2011;24(7):816-821.

33. Morosetti M, Gorini A, Costanzo AM. Clinical management of nondialysis patients with chronic kidney disease: a retrospective observational study. Data from the SONDA study (Survey Of Non-Dialysis outpAtients). Int $J$ Nephrol Renovasc Dis. 2013;6: $27-37$.

\section{Publish your work in this journal}

The International Journal of Nephrology and Renovascular Disease is an international, peer-reviewed open-access journal focusing on the pathophysiology of the kidney and vascular supply. Epidemiology, screening, diagnosis, and treatment interventions are covered as well as basic science, biochemical and immunological studies. The journal welcomes original research, clinical studies, reviews \& evaluations, expert opinion and commentary, case reports and extended reports. The manuscript management system is completely online and includes a very quick and fair peerreview system, which is all easy to use. Visit http://www.dovepress.com/ testimonials.php to read real quotes from published authors. 